\title{
Reduction of Atmospheric Effects in Satellite Images During the COVID-19 Induced Lockdown
}

\author{
Ashish Joshi $^{1} \cdot$ Shefali Agrawal ${ }^{2} \cdot$ Prakash Chauhan $^{3}$
}

Received: 25 June 2020 / Accepted: 17 September 2020 / Published online: 29 September 2020

(C) Indian Society of Remote Sensing 2020

\begin{abstract}
The quality of satellite images is affected due to the scattering and absorption of sunlight by atmospheric molecules and aerosols. Aerosols are small particles suspended in the atmosphere which affects the air quality. The concentration and characteristics of the atmospheric aerosols can vary from place to place. Aerosols are major constituents for the formation of haze and thus responsible for degradation in the satellite images. During the novel corona virus disease pandemic (COVID-19) countrywide lockdown in India, aerosols level significantly lowered in the north Indian cities in the IndoGangetic plain. In this paper, an analysis of image quality was done for Sentinel-2A/2B satellite images for different north Indian cities with respect to the impact of aerosols on image sharpness and surface reflectance values during the prelockdown and lockdown period. The image quality of the images was evaluated through the power spectrum, gradient, and reflectance values in the image. It was observed that the sharpness of the satellite images increased during the lockdown period due to the reduction in aerosols levels in the atmosphere. There was an increase in the power spectrum values from $5 \%$ (minimum) to $12 \%$ (maximum) in the blue band, $4 \%$ (minimum) to $10 \%$ (maximum) in the green band, and $1.7 \%$ (minimum) to $6 \%$ (maximum) in the red band in the satellite images of major cities in the Indo-Gangetic plain during the lockdown period. Since haze is an additive component in the images, thus it was also observed that surface reflectance values had decreased in visible bands for the features which have low surface reflectance values like water (30\%), vegetation (21\%) and barren land (17\%) and surface reflectance values had increased in visible bands for the features which have high surface reflectance values like building (19\%) during the lockdown period.
\end{abstract}

Keywords Power spectrum $\cdot$ COVID-19 $\cdot$ Surface reflectance $\cdot$ Sentinel-2A/2B $\cdot$ AOD $\cdot$ Lockdown

Ashish Joshi

ashish@iirs.gov.in

Shefali Agrawal

shefali_a@iirs.gov.in

Prakash Chauhan

prakash@iirs.gov.in

1 Geospatial Technology and Outreach Programme Group, Photogrammetry and Remote Sensing Department, Indian Institute of Remote Sensing, Indian Space Research Organisation (ISRO), Dehradun, India

2 Geospatial Technology and Outreach Programme Group, Indian Institute of Remote Sensing, Indian Space Research Organisation (ISRO), Dehradun, India

3 Indian Institute of Remote Sensing, Indian Space Research Organisation (ISRO), Dehradun, India

\section{Introduction}

Sharpness in the image can be defined by the frequency content in the image. Aerosols, major contributors in the haze, generally reduce the sharpness in the image (Kopeika et al. 1998; Yoram 1984). India observed the nationwide lockdown from 25/03/2020 due to COVID-19 pandemic. Due to a reduction in the functioning of the industries and vehicles during the lockdown period, atmospheric pollutants like aerosols, $\mathrm{NO}_{2}, \mathrm{CO}$, etc., had significantly reduced (Dutheil et al. 2020). Due to a reduction in the atmospheric pollutants, the haze in the major cities was significantly reduced during the lockdown period. Improvement in the air quality in the major cities in China during the COVID19 pandemic has been analysed in the paper by Guojun et al. (2020).Also, improvement in the air quality during 
the COVID-19 pandemic has been recently analysed in the paper by Ranjana et al. (2020).

The atmosphere in the Indo-Gangetic plain is often contaminated with aerosols due to dense population and economic activities. Aerosol optical depth (AOD) measures the quantitative estimate of the aerosol present in the atmosphere. The analysis of the aerosol optical thickness over land, snow, and sea is explained in the paper by Sifakis (2000). The aerosol optical depth of less than 0.1 over the atmosphere is an indicator of the clean atmosphere. The aerosols level monitored through Spaceborne Moderate Resolution Imaging Spectroradiometer (MODIS) on NASA's Terra satellite for pre-lockdown and lockdown period was analysed. The Aerosol Level-3 product at $550 \mathrm{~nm}$ of MODIS Terra satellite was acquired from 21/03/ 2020 to 23/03/2020 (pre-lockdown period) and 28/03/2020 to $30 / 03 / 2020$ (lockdown period) has been used for analysis. It was observed that the AOD level significantly decreased from 0.6 in the pre-lockdown to 0.2 in the lockdown period in most of the major cities in the IndoGangetic plain. Aerosol optical depth level for date 22/03/ 2020 and 30/03/2020 is shown in Fig. 1.

Aerosols contribute to atmospheric scattering and absorption in the visible and NIR spectrum, thereby degrading satellite image quality and can significantly affect remote sensing applications such as change detection, classification, and target recognition. The study focusses on the evaluation of the satellite image quality with respect to the effect on image sharpness and reflectance values for different features in different cities of the Indo-Gangetic plain in north India for pre-lockdown and lockdown period due to reduction in aerosol levels. The image quality has been quantified by calculating the power spectrum, gradient, and spectral response of different features in the image.

\section{Datasets}

Sentinel-2A/2B level-1C images acquired for the cities of New Delhi, Agra, Patna, and Varanasi in the Indo-Gangetic plain are used for image quality assessment during the lockdown period. The Sentinel-2 mission comprises of a constellation of two polar-orbiting satellites (Sentinel-2A/ 2B) placed in the same sun-synchronous orbit, phased at $180^{\circ}$ to each other (Gatti and Bertolini 2015). The constellation aims for observing the changes in the land surface condition with high revisit time (10 days at the equator with one satellite, and 5 days with 2 satellites). The detailed information of Sentinel-2A/2B different bands is described in Table 1.

The cloud-free Sentinel-2A/2B datasets have been used for image quality assessment between 01/03/2020 and $25 / 03 / 2020$ for the pre-lockdown period and between $25 / 03 / 2020$ and $05 / 04 / 2020$ for the lockdown period. The details of the datasets are given in Table 2 .

The natural colour composite (NCC) images using band 4 (red), band 3 (green), and band 2 (blue) for New Delhi, Agra, Patna, and Varanasi city were extracted and are shown in Fig. 2. In the visual examination, the images acquired during the lockdown period are looking sharper than the pre-lockdown period due to non-hazy conditions.

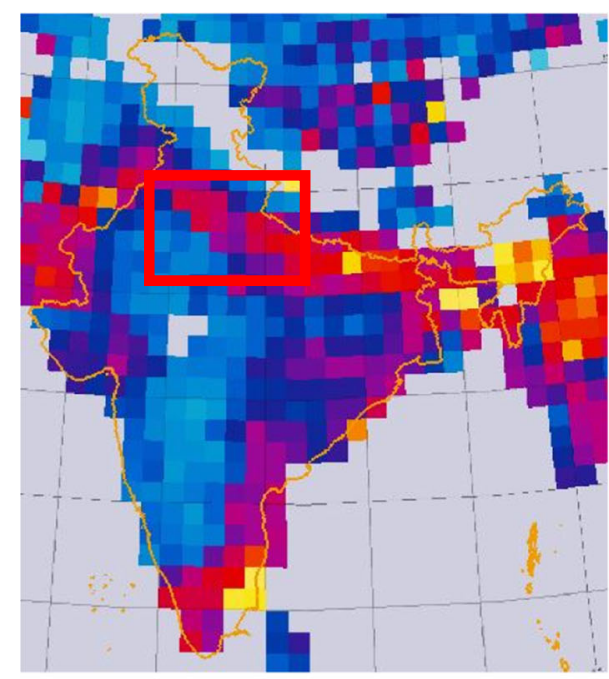

23/03/2020 (Pre-Lockdown)

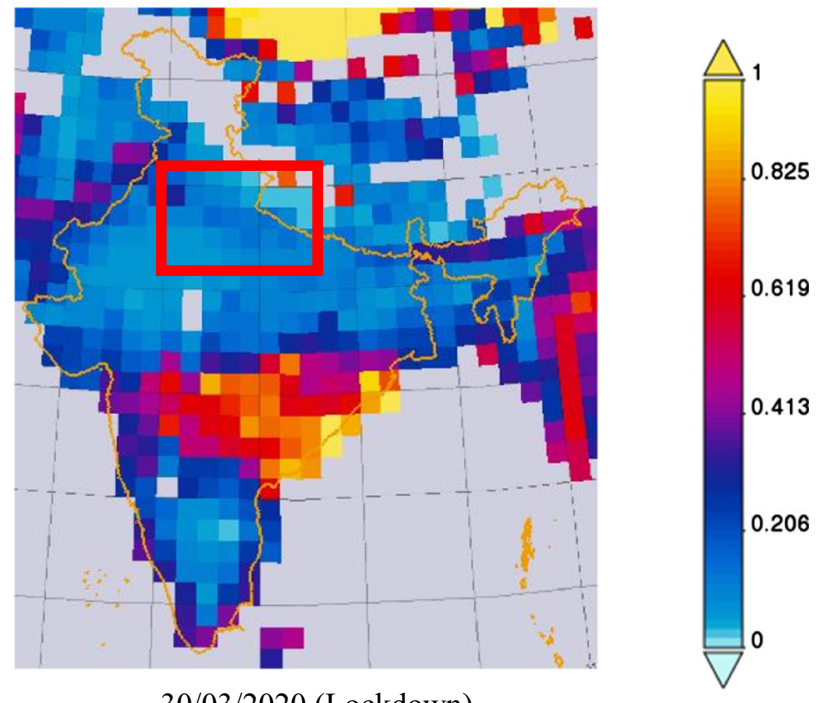

30/03/2020 (Lockdown)

Fig. 1 Aerosol optical depth $(550 \mathrm{~nm})$ measured through MODIS over Indian region. Data source: https://giovanni.gsfc.nasa.gov/giovanni/ 
Table 1 Sentinel-2A/2B multispectral band description

\begin{tabular}{lll}
\hline Sentinel-2A/2B bands & Central wavelength $(\mu \mathrm{m})$ & Resolution $(\mathrm{m})$ \\
\hline Band-1 coastal aerosols & 0.443 & 60 \\
Band-2 blue & 0.490 & 10 \\
Band-3 green & 0.560 & 10 \\
Band-4 red & 0.665 & 10 \\
Band-5 vegetation red edge & 0.705 & 20 \\
Band-6 vegetation red edge & 0.740 & 20 \\
Band-7 vegetation red edge & 0.783 & 20 \\
Band-8 NIR & 0.842 & 10 \\
Band-8A vegetation red edge & 0.865 & 20 \\
Band-9 SWIR-cirrus & 0.945 & 60 \\
Band-10 SWIR & 1.375 & 60 \\
Band-11 SWIR & 1.610 & 20 \\
Band-12 SWIR & 2.190 & 20
\end{tabular}

Table 2 Details of sentinel-2A/2B datasets in the present study

\begin{tabular}{lccc}
\hline City & Satellite & Date of pass & Datasets \\
\hline New & Sentinel- & $10 / 03 / 2020,30 / 03 / 2020,16 / 03 /$ & S2B_MSIL1C_20200310T052649_N0209_R105_T43RGM_20200310T090919, \\
Delhi & 2B & 2019,31/03/2019 & S2B_MSIL1C_20200330T052649_N0209_R105_T43RGM_20200330T090926, \\
& & & S2B_MSIL1C_20190316T052649_N0207_R105_T43RGM_20190316T105044, \\
& & & S2A_MSIL1C_20190331T052651_N0207_R105_T43RGM_20190331T091644 \\
Agra & Sentinel- & 02/03/2020, 04/04/2020,01/03/ & S2A_MSIL1C_20200302T051721_N0209_R062_T43RGL_20200302T085915, \\
& 2A & 2019,10/04/2019 & S2A_MSIL1C_20200404T052641_N0209_R105_T43RGL_20200404T082606, \\
& & & S2A_MSIL1C_20190301T052741_N0207_R105_T43RGL_20190301T090929, \\
Patna & Sentinel- & 03/03/2020, 02/04/2020 & S2A_MSIL1C_20190410T052651_N0207_R105_T43RGL_20190410T091939 \\
& 2A & & S2A_MSIL1C_20200303T044701_N0209_R076_T45RUJ_20200303T075549, \\
Varanasi & Sentinel- & 16/03/2020,05/04/2020 & S2A_MSIL1C_20200402T044701_N0209_R076_T45RUJ_20200402T074737 \\
& 2A & & S2A_MSIL1C_20200316T045651_N0209_R119_T44RPP_20200316T084527, \\
& & S2A_MSIL1C_20200405T045651_N0209_R119_T44RPP_20200405T080201 \\
\hline
\end{tabular}

\section{Methodology}

To quantify the image quality, sharpness, and reflectance values (TOA) are analysed for all the images. The sharpness of the image is evaluated through the power spectrum method and the gradient method. The reflectance values are evaluated through spectral curves. The details of the different methods adopted for image quality assessment are described in the following sections.

\section{Power Spectrum Method}

The image that is focussed has a sharper edge. The edge information corresponds to the higher value in the frequency domain and hence, the image power spectrum can be used to represent the image definition. Since haze in the image acts as an additive component, thus edges of the images are blurred resulting in the decrease in the values of the power spectrum. In this method, the power spectrum of the image is calculated and then the average power spectrum of the image is calculated at the Nyquist frequency. The method of estimating the power spectrum of the image is explained in the papers by Michael and Brian (2019) and Eriksson (2000). Sharp images have higher values of the power spectrum in the image. If $f(x, y)$ represents the image then the power spectrum of the image is defined as Power $\operatorname{Spectrum}(u, v)=|H(u, v)|^{2}$

where $H(u, v)$ represents the image in the frequency domain.

In the $\mathrm{dB}$ scale,

Power $\operatorname{Spectrum}(u, v)=10 * \log 10\left(|H(u, v)|^{2}\right)$

The flowchart of the method is shown in Fig. 3. 


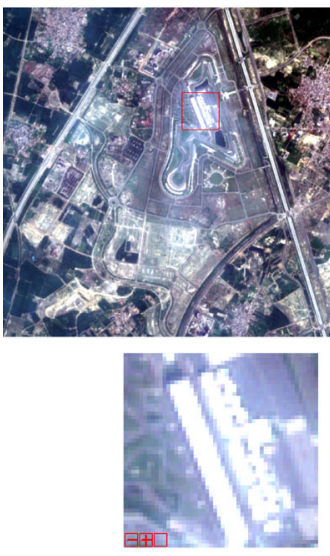

$10 / 03 / 2020$

(a) Sentinel-2B image of New Delhi during pre-lockdown and lockdown period
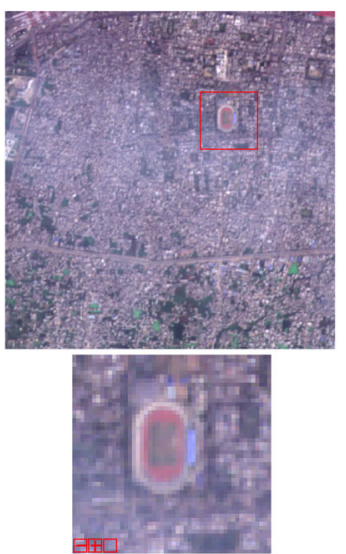

$03 / 03 / 2020$
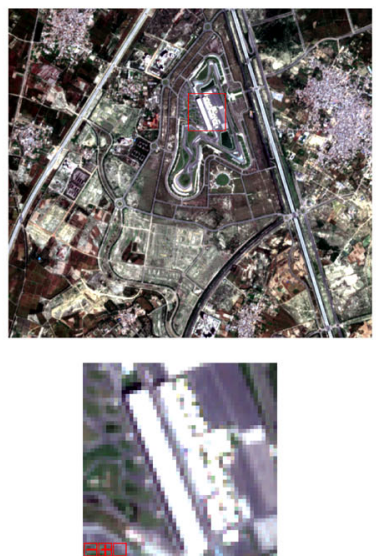

$30 / 03 / 2020$

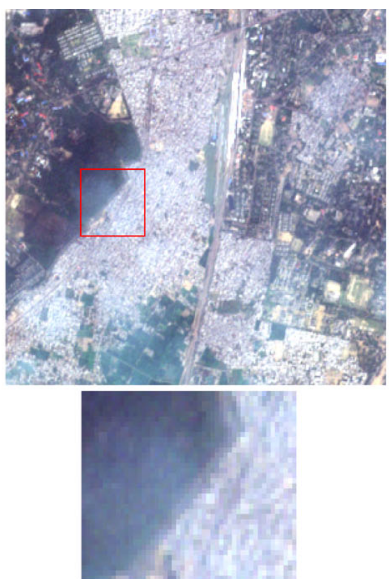

$02 / 03 / 2020$
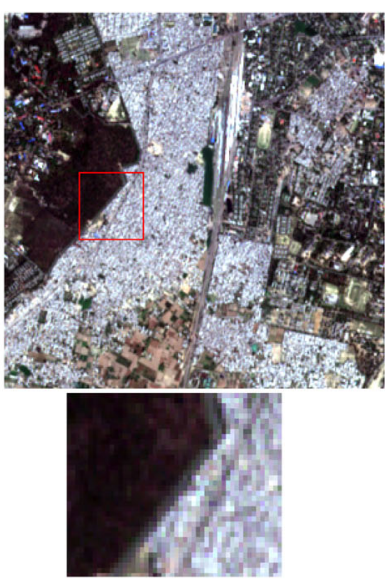

$04 / 04 / 2020$ (b) Sentinel-2A image of Agra during pre-lockdown and lockdown period
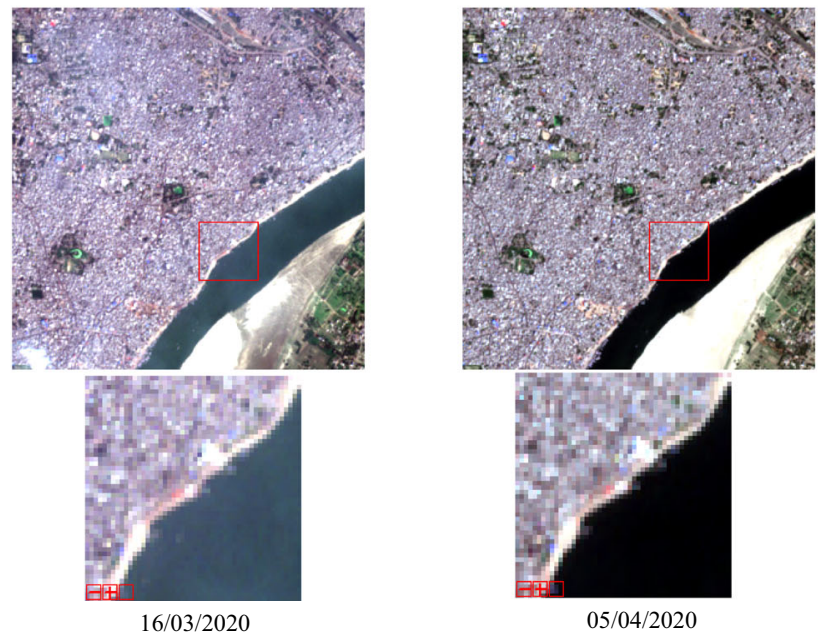

(d) Sentinel-2A image of Varanasi during Pre-lockdown and lockdown period

Fig. 2 Sentinel-2 images of different cities during pre-lockdown and lockdown

\section{Gradient Method}

The gradient of the image is a powerful method to find the edges in the images. Gradient-based edge detection is analysed in the paper by Zenzo (1986). Edges generally define the high-frequency component in the images. Thus, the gradient of the image can be used to quantify the image with respect to the sharpness of the image.

In this method, the gradient of the image is calculated in the scan and pixel direction in the image. The histogram of the gradient values is computed for both directions. Sharp images have higher gradient values in the image. If $f(x, y)$ represents the image then the gradient in $\mathrm{x}$ (pixel) and $\mathrm{y}$ (scan) direction is defined as

$$
\begin{aligned}
& f_{x}=\frac{\partial f(x, y)}{\partial x} \\
& f_{y}=\frac{\partial f(x, y)}{\partial y}
\end{aligned}
$$

The flowchart of the method is shown in Fig. 4.

\section{Reflectance Method}

Spectral reflectance is a measure of energy that a surface reflects at a specific wavelength. Many surfaces reflect the different amounts of energy in different portions of the spectrum. Haze in the image contaminates the visible band and thus changes the reflectance values of the features. Thus, image quality is assessed by measuring the reflectance values of the hazy pixels of different features. In this 


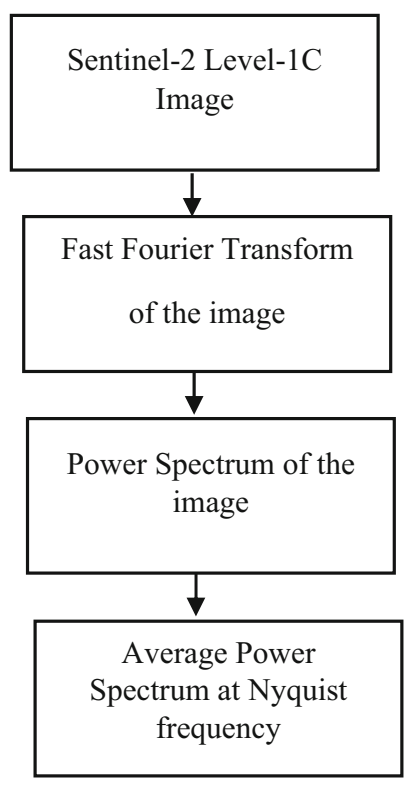

Fig. 3 Flowchart for power spectrum estimation method

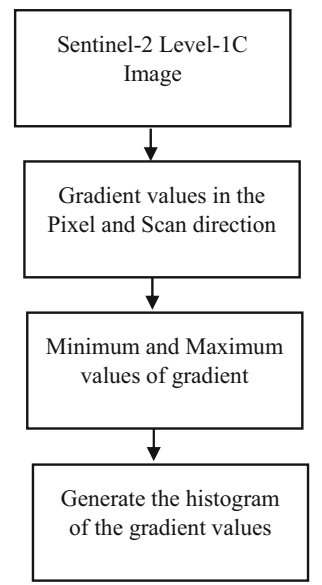

Fig. 4 Flowchart for gradient estimation method

method, the mean reflectance of the image has been calculated for a particular window $\left(k^{*} k\right)$ around the desired pixel. The reflectance of the features is calculated for each red, green, and blue band. The flowchart of the method is shown in Fig. 5.

The spectral curve represents the reflectance values of a particular feature at different wavelengths. Spectral responses of the different targets were given in the NASA technical report by Bowker et al. (1985). Since haze and aerosols change the reflectance values of the pixels, thus spectral curve is used to assess the quality of images. The flowchart of estimating the spectral curve is shown in Fig. 6.

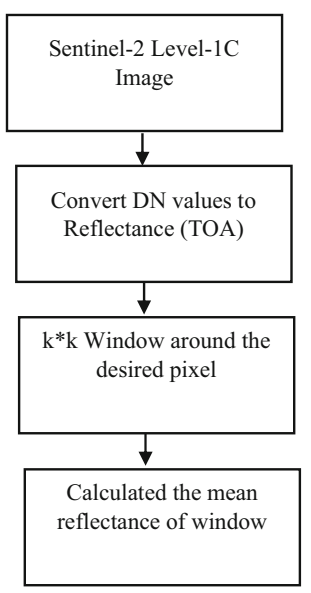

Fig. 5 Flowchart for reflectance calculation method

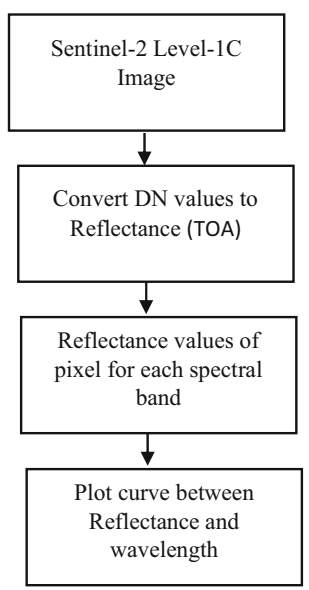

Fig. 6 Flowchart for spectral curve method

\section{Results}

Image quality assessment of Sentinel-2A/2B images of different Indian cities for pre-lockdown and lockdown period was done by evaluating the power spectrum, gradient, and surface reflectance (TOA) to analyse the reduction in atmospheric effects in the satellite images.

\section{Power Spectrum Method}

The power spectrum for satellite images of all cities is estimated at Nyquist frequency. It is observed that the values of the power spectrum of the satellite images have increased during the lockdown period as compare to the pre-lockdown period, which increases the sharpness of the images. Since haze mostly affects the image in the visible spectrum, thus this method has been applied for the red, blue, and green band for image quality assessment. Table 3 
Table 3 Power spectrum values of images for different cities

\begin{tabular}{|c|c|c|c|c|c|c|c|c|c|}
\hline \multirow[t]{2}{*}{ Frequency power spectrum $(\mathrm{dB})$} & \multicolumn{3}{|c|}{ Pre-lockdown period } & \multicolumn{3}{|c|}{ Lockdown period } & \multicolumn{3}{|c|}{ Percentage change } \\
\hline & Red & Green & Blue & Red & Green & Blue & Red & Green & Blue \\
\hline New Delhi & -63.5 & -67.0 & -70 & -59.7 & -61.7 & -63.4 & 5.98 & 7.91 & 9.42 \\
\hline Agra & -68.8 & -73.1 & -75 & -64.4 & -65.6 & -66.4 & 6.39 & 10.25 & 12.40 \\
\hline Patna & -69.4 & -73.8 & -76 & -67.3 & -69.8 & -72.0 & 3.02 & 5.42 & 6.37 \\
\hline Varanasi & -62.5 & -66.2 & -69 & -61.4 & -63.4 & -65.1 & 1.76 & 4.22 & 5.65 \\
\hline
\end{tabular}
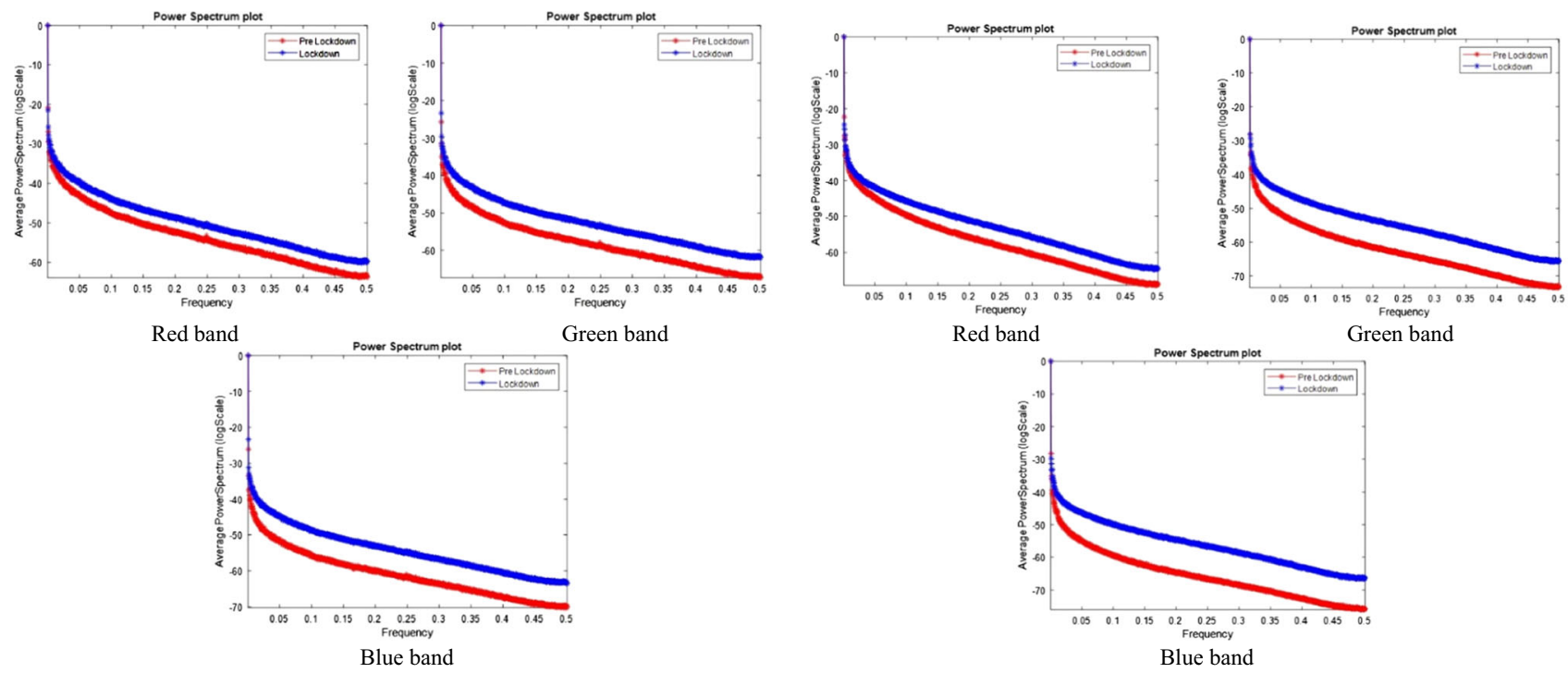

(a) Power spectrum plot for New Delhi image

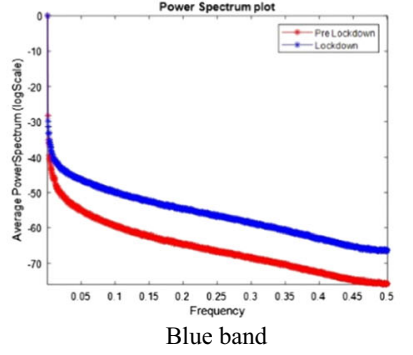

(b) Power spectrum plot for Agra image
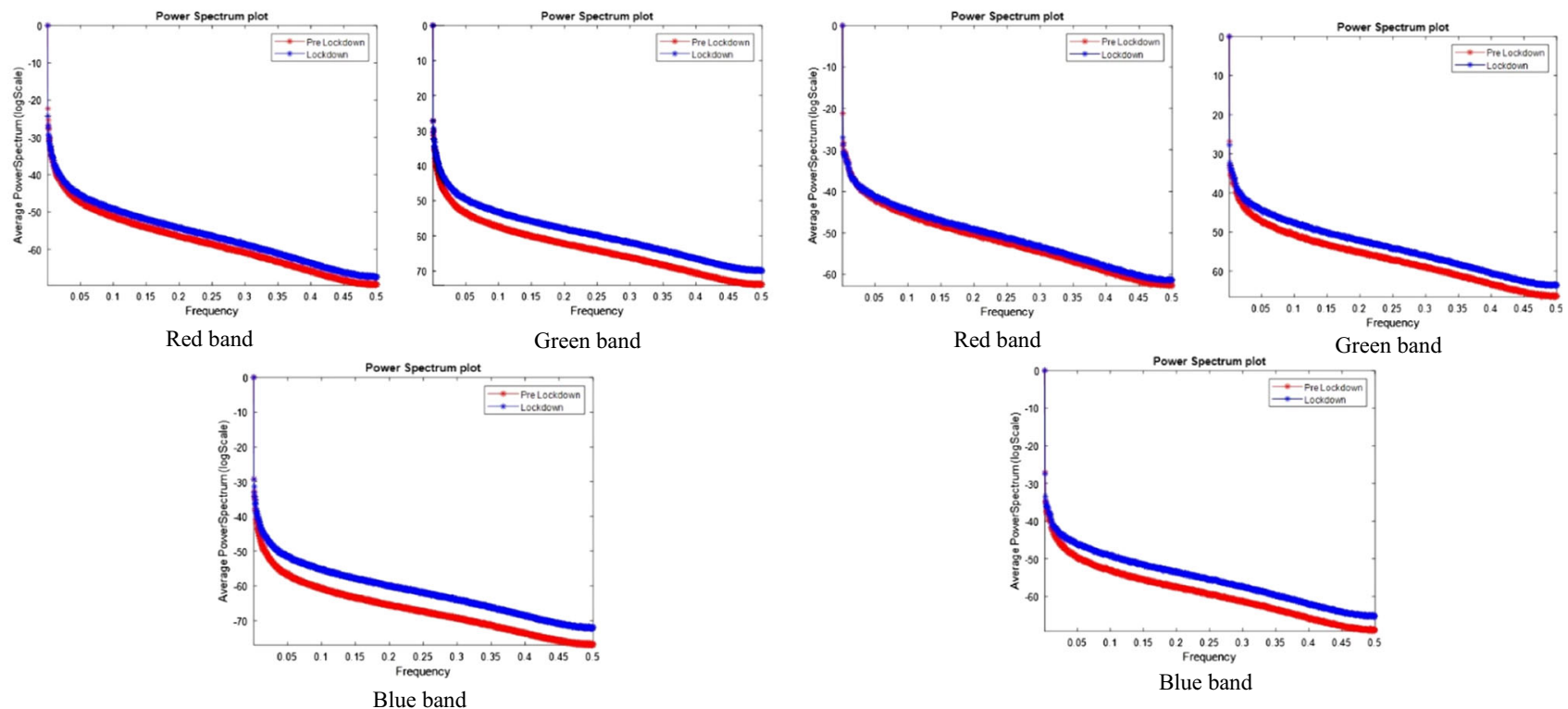

(c) Power spectrum plot for Patna image

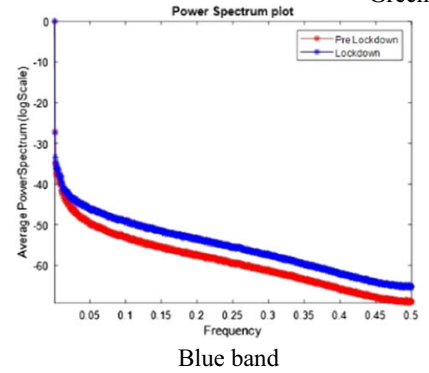

(d) Power spectrum plot for Varanasi image

Fig. 7 Power spectrum plot for images of different cities during pre-lockdown and lockdown period 
shows that the percentage change of the power spectrum values in the New Delhi image is $5.98 \%$ for the red band, $7.91 \%$ for the green band, and $9.42 \%$ for the blue band. In the Agra image, the percentage change is $6.39 \%$ for the red band, $10.25 \%$ for the green band, and $12.4 \%$ for the blue band. In the Patna image, the percentage change is $3.02 \%$ for the red band, $5.42 \%$ for the green band, and $6.37 \%$ for the blue band. In the Varanasi image, the percentage change is $1.76 \%$ for the red band, $4.22 \%$ for the green band, and $5.65 \%$ for the blue band. The improved power spectrum values in the images show the enhancement of the frequency content and thus image quality was improved during the lockdown period. It was observed that the sharpness has increased more in the blue band as compared to the red band and green band during the lockdown period which indicates that the reduction in the atmospheric effects in the images because the blue band is mostly affected by the atmospheric conditions.

The power spectrum profile of the different cities is shown in Fig. 7 in which red colour is for pre-lockdown period and blue colour is for the lockdown period. The power spectrum shows that increase in the power spectrum at all frequencies is highest in the blue band as compared to green and red bands for all the cities during the lockdown period. Since the blue band is more affected by the atmospheric conditions thus an improvement in the power spectrum of the blue band indicates a reduction in the atmospheric effects in the images.

The pre-lockdown and lockdown period was also the transition period from the Rabi season to the summer season. Thus, to decouple the effect of atmospheric effects from surface influence, the power spectrum of the band 1 (ultra-blue) and band 8 (near infra-red) is analysed. Band 1 of Sentinel-2A/2B is mainly affected by atmospheric constituents, whereas band 8 is less affected by atmospheric constituents. Figure 8 shows that the image quality of the ultra-blue band (band 1) for New Delhi and Agra image is much improved during the lockdown period as compared to the band 8 (near infra-red) image.

Figure 9 shows the power spectrum of the band 1 and band 8 for the New Delhi and Agra image. On analysis of the power spectrum of the image of New Delhi and Agra, it is observed that the power spectrum of the band-1 (ultrablue) has significantly improved during the lockdown period as compare to the pre-lockdown period. However, the power spectrum of the band-8 (NIR) is less affected during the lockdown period as compare to the pre-lockdown period. Thus, it shows that improvement in the satellite images in the lockdown period was mainly due to improvement in the atmospheric conditions as compare to surface conditions. Figure 9 shows the power spectrum profile of the band 1 and band 8 of the New Delhi and Agra image during the pre-lockdown and lockdown image. In

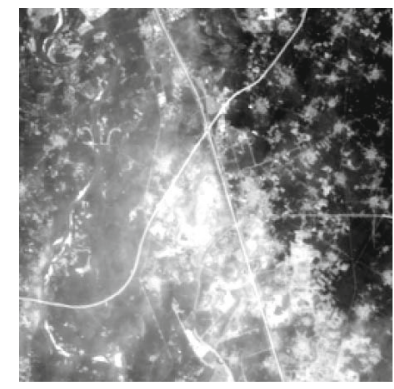

Ultra-blue band (10/03/2020)

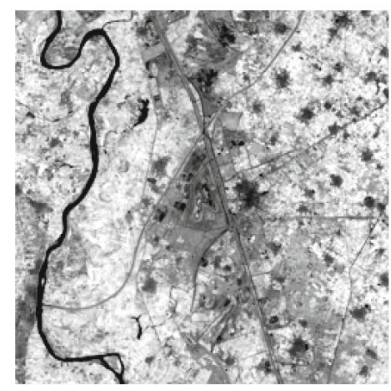

NIR band (10/03/2020)

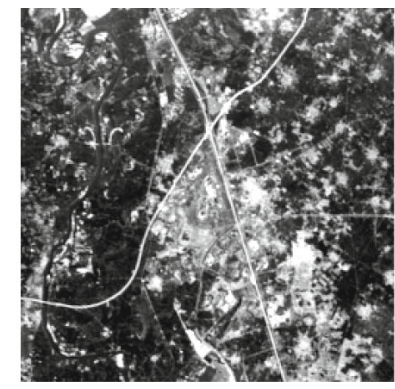

Ultra-blue band (30/03/2020)

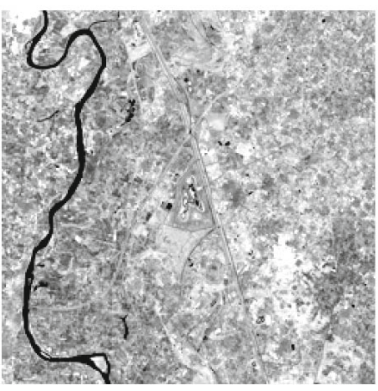

NIR band $(30 / 03 / 2020)$

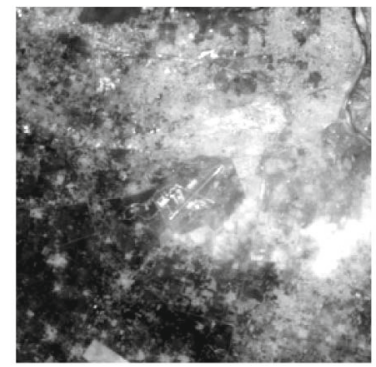

Ultra-blue band (02/03/2020)

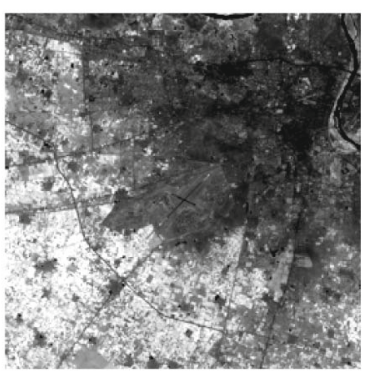

NIR band $(02 / 03 / 2020)$

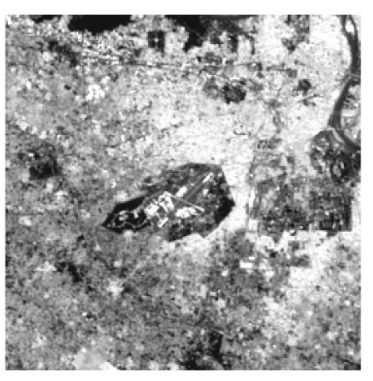

Ultra-blue band (04/04/2020)

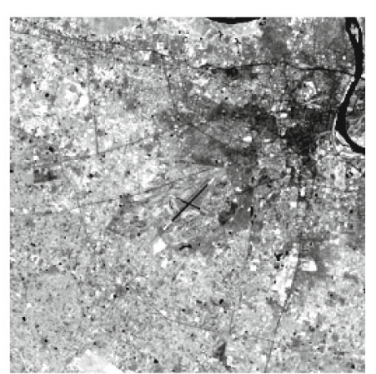

NIR band (04/04/2020)

(a) New Delhi Images during pre-lockdown and lockdown period

Fig. 8 Ultra-blue and NIR band images of New Delhi and Agra during pre-lockdown and lockdown period 


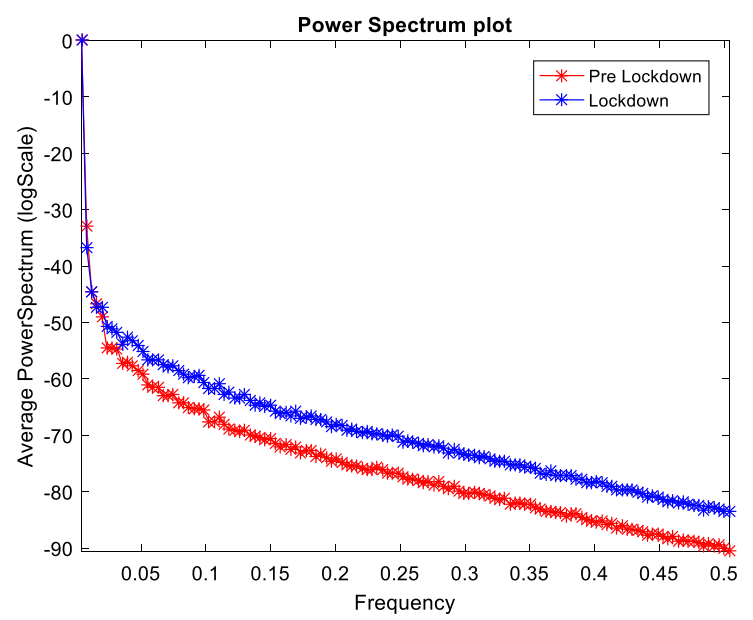

Ultra-blue band

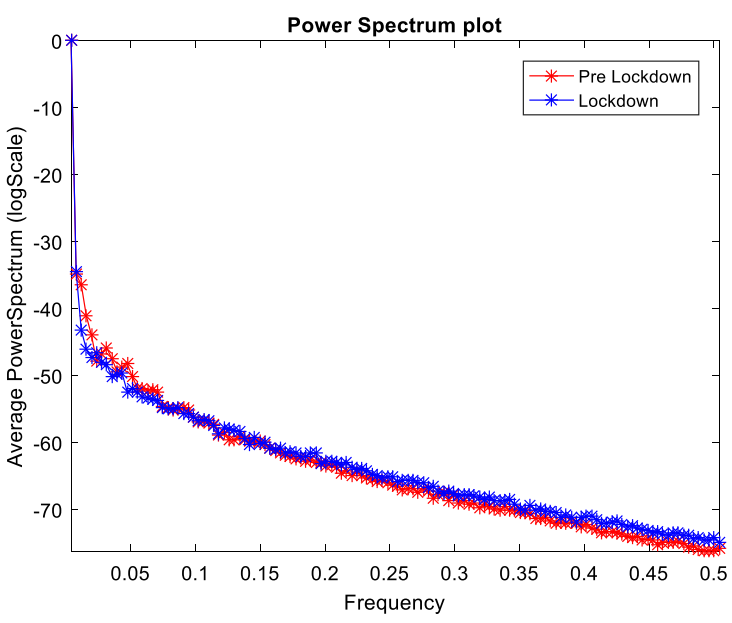

NIR band

(a) Power spectrum profile for New Delhi

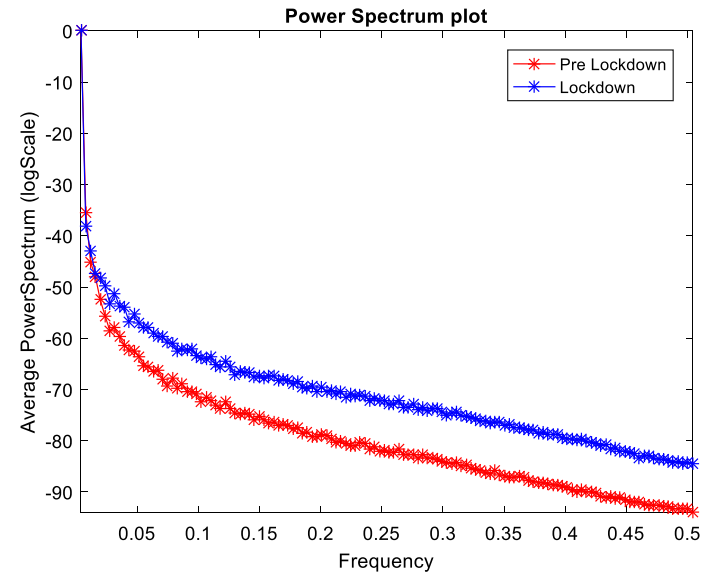

Ultra-blue band

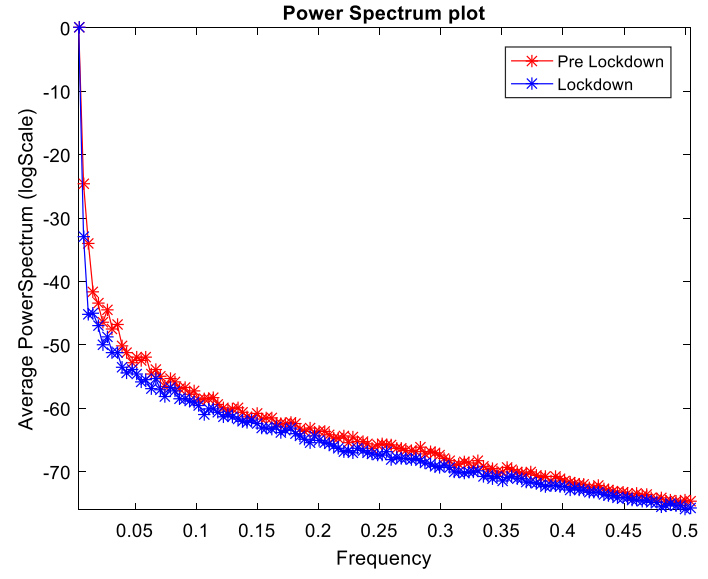

NIR band

(b) Power spectrum profile for Agra

Fig. 9 Ultra-blue and NIR band power spectrum profile of New Delhi and Agra during pre-lockdown and lockdown period

Fig. 9, the red colour is for the pre-lockdown period and the blue colour is for the lockdown period.

Comparative analysis of the power spectrum is also done for the datasets of similar dates for the year 2020 and 2019 for New Delhi and Agra image. From Fig. 10, it can be inferred that the power spectrum of the blue band for New Delhi and Agra images is higher during the lockdown period in the year 2020 as compared to a similar date in the year 2019. Thus, the blue band shows significant improvement during the lockdown period in the year 2020 as compared to the similar dates in the year 2019 for the New Delhi and Agra images. Since atmospheric conditions mainly affect the blue band thus it can be concluded that the effect of atmospheric conditions is reduced in the satellite images for the lockdown period of the year 2020 as compared to the year 2019. In Fig. 10, the blue colour represents the power spectrum in the year 2020 and the red colour represents the power spectrum in the year 2019.

Figures 11 and 12 show that images of the New Delhi and Agra in the blue band are significantly improved in the lockdown period in the year 2020 as compared to similar dates in the year 2019.

\section{Gradient Method}

Image quality is also quantified by applying the gradient method on the images of the pre-lockdown and lockdown period. The histogram of the gradient values is computed between the minimum and maximum gradient values for all images of different cities. In Fig. 13, the shape of the gradient plot in the lockdown period is broad due to an increase in the frequency content, whereas the shape of the 


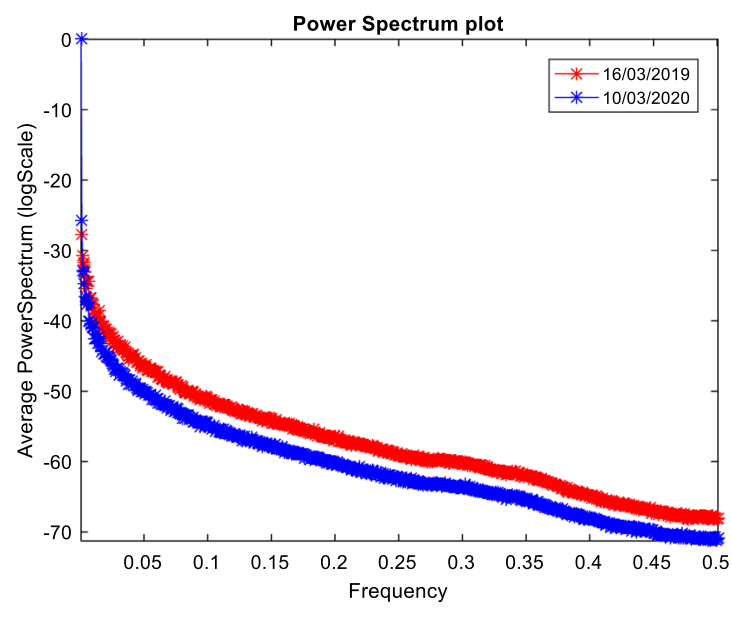

$16 / 03 / 2019 \& 10 / 03 / 2020$

(Pre-lockdown period)

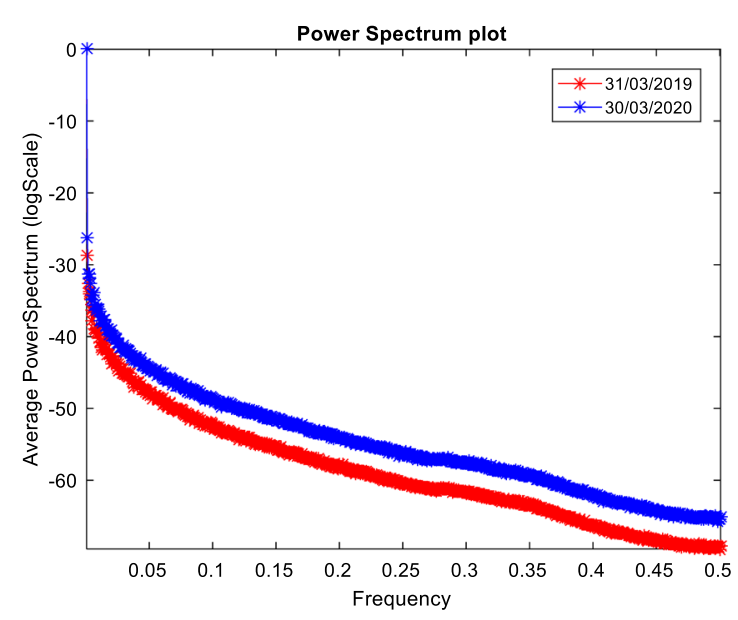

$31 / 03 / 2019 \& 30 / 03 / 2020$

(Lockdown period)

(a) Power spectrum profile for New Delhi

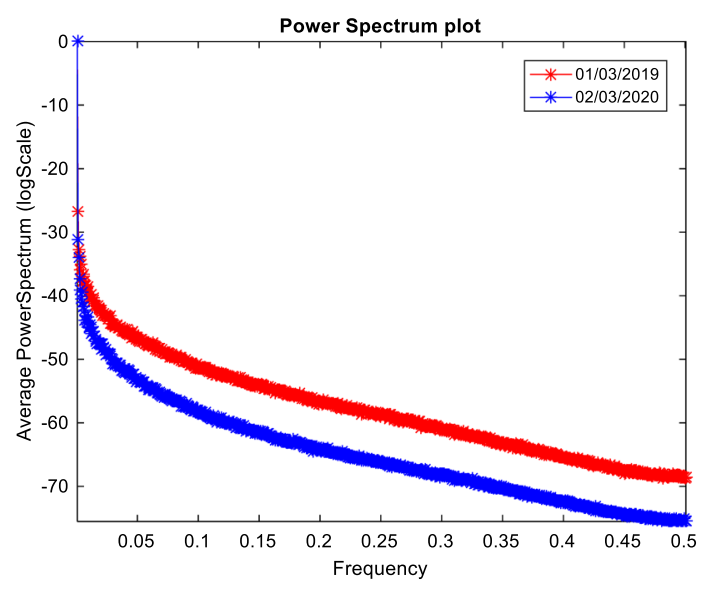

01/03/2019\&02/03/2020

(Pre-lockdown period)

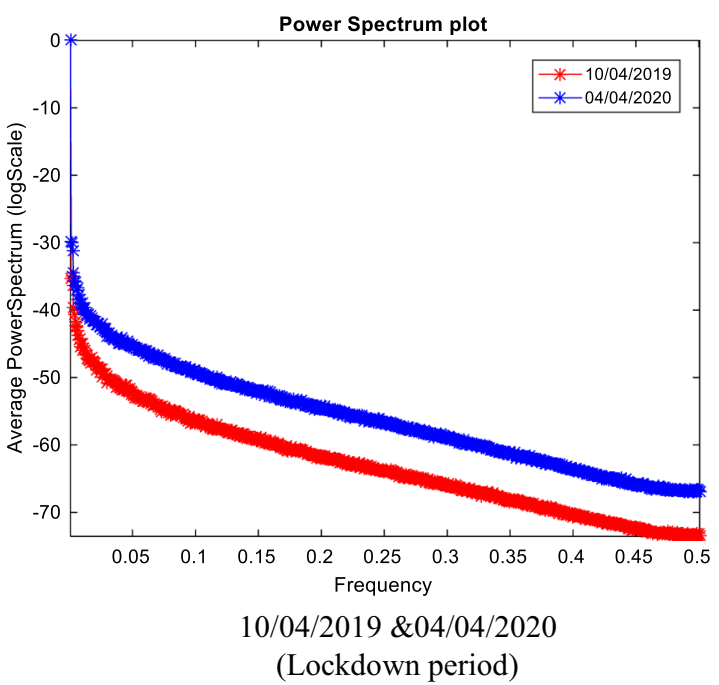

(Lockdown period)

(b) Power spectrum profile for Agra

Fig. 10 Power spectrum profile for blue band of New Delhi and Agra during pre-lockdown and lockdown period in 2020 and similar dates in 2019

gradient plot in the pre-lockdown period is narrow due to less frequency content as compare to the lockdown period. Thus, it is observed from the histogram plot that the gradient values have increased in the images acquired during the lockdown period due to an increase in frequency content in the image. The observations are similar as observed with the power spectrum plot. It is also observed the change is higher in the blue band as compared to the red band and green band which shows that sharpness in the blue band has increased and observations are similar to the power spectrum analysis. It can be concluded from the gradient method analysis that image quality is improved due to a reduction in the atmospheric effects during the lockdown period. In Fig. 13, the red colour is for the prelockdown period and the blue colour is for the lockdown period in the histogram plot of gradient values.

\section{Reflectance Method}

The reflectance values are calculated for different features like building, water, vegetation, and barren field for each of the visible bands in images of different cities acquired during pre-lockdown and lockdown period. Then, the percentage change of reflectance in each of the visible 


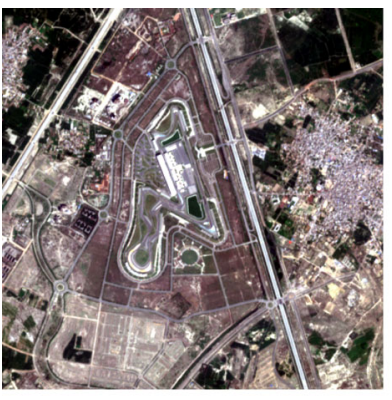

$16 / 03 / 2019$

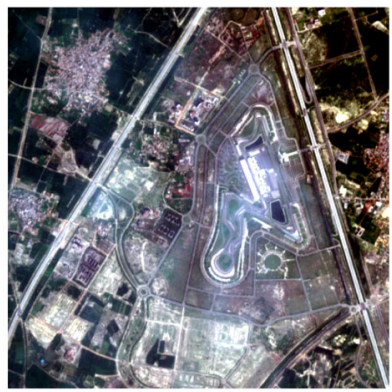

10/03/2020 (Pre-lockdown)

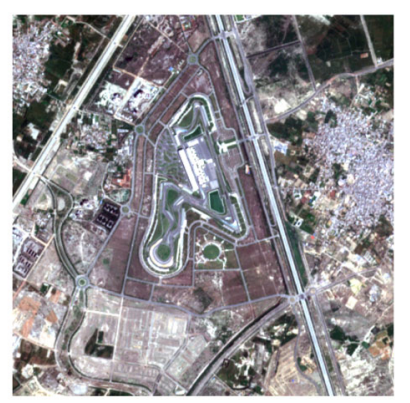

$31 / 03 / 2019$

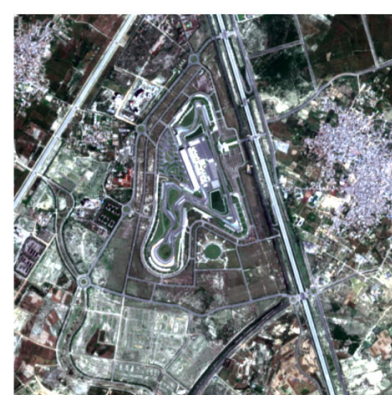

30/03/2020 (Lockdown)
Fig. 11 New Delhi image during pre-lockdown and lockdown period in 2019 and 2020

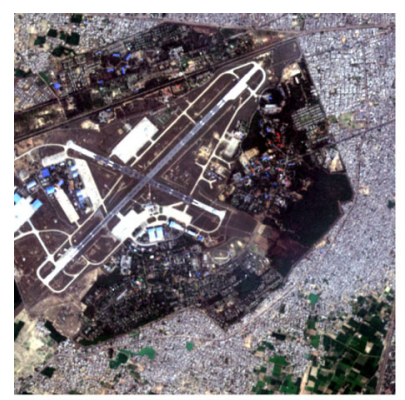

$01 / 03 / 2019$

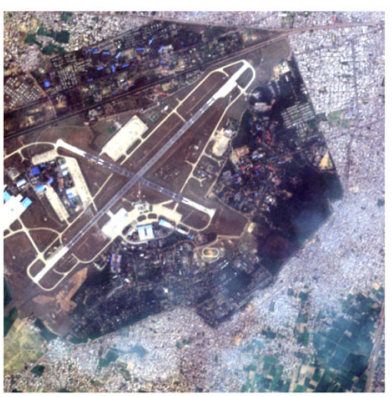

02/03/2020 (Pre-lockdown)

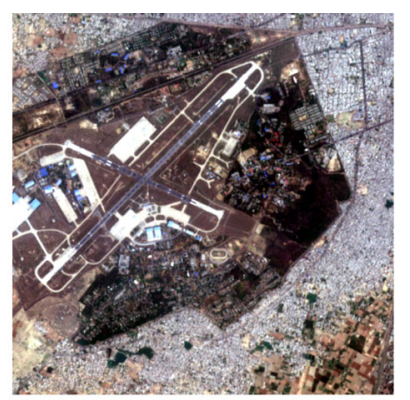

$10 / 04 / 2019$

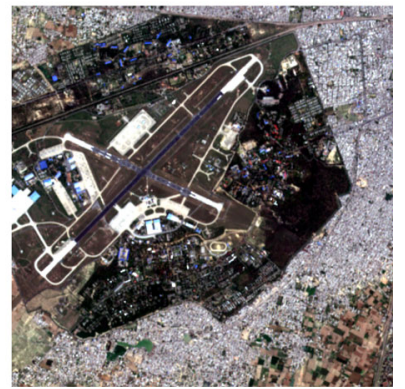

04/04/2020 (Lockdown)
Fig. 12 Agra image during pre-lockdown and lockdown. Period in 2019 and 2020

bands (red, green and blue) is calculated. The average percentage change is then calculated by taking an average of the percentage change in each of the visible band. The reflectance values and average percentage change of different features in different cities during pre-lockdown and lockdown period are shown in Table 4. The digital number values $(\mathrm{DN})$ in the level-1C product of the Sentinel-2 images is converted into reflectance values (TOA) by dividing with the quantification factor $(Q)$,

Reflectance $=\frac{\mathrm{DN}}{Q}$

where for Sentinel-2A/2B, $Q=10,000$.

Table 4 shows that there is a decrease in the reflectance values for vegetation, barren land, and water (low reflectance values) during the lockdown period due to a reduction in atmospheric effects in the images. It is observed that surface reflectance values have decreased during the lockdown period in visible bands for the features which have low surface reflectance values like water $(30 \%)$, vegetation $(21 \%)$, and barren land $(17 \%)$ because atmospheric constituents like haze act as an additive component for the low reflectance features (water, vegetation, and barren land) and thus due to reduction in the atmospheric effects, the reflectance is also decreased. However, surface reflectance values have increased during the lockdown period in visible bands for the features which have high surface reflectance values like building (19\%) because atmospheric constituents do not affect much on high reflectance features like buildings as they have much higher reflectance values. Since the blue band is affected more with haze so more changes are observed in the blue band. Thus, the contrast in the images has increased during the lockdown period due to a decrease in the reflectance values of the low reflectance features (water, vegetation, and barren land) and an increase in the reflectance values of the high reflectance features (building and structures). As it is a known fact that the contrast increases with the increase in the frequency content of the image and thus, reflectance values analysis shows a similar trend with the power spectrum analysis.

The spectral curves of different features are plotted from the images of different cities for the entire spectrum. Most of the features are affected due to haze during pre-lockdown and changes are observed in reflectance values in the visible band of the spectral curves during pre-lockdown and lockdown period. Spectral curves of different features are shown in Fig. 9. The red colour is for the pre-lockdown period and the blue colour is for the lockdown period in the spectral curves of urban building, vegetation, barren land, and water in Fig. 14.

\section{Conclusions}

During the nation-wide lockdown due to COVID-19 in India, aerosols level significantly decreased in the cities of Indo-Gangetic plain. Due to a reduction in aerosols level, 

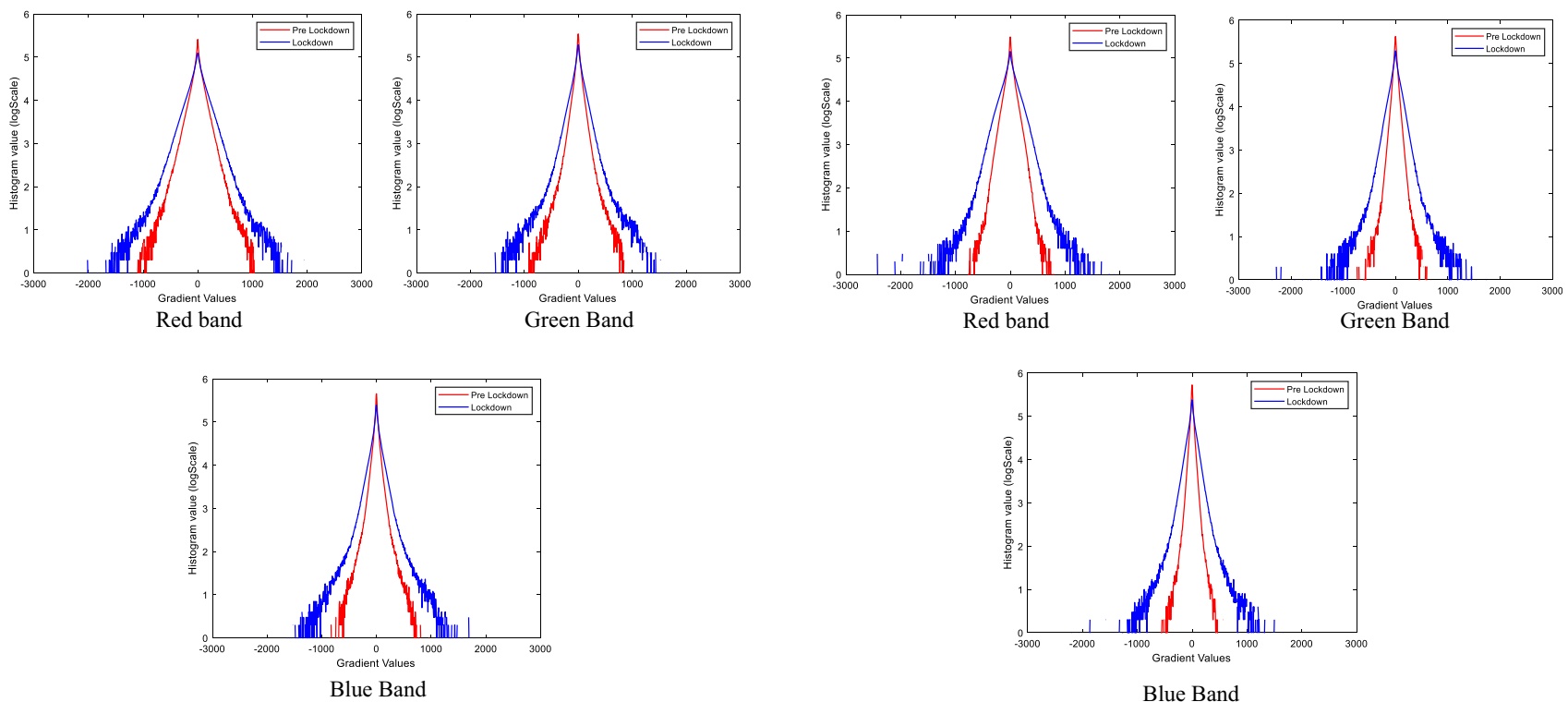

(a) Histogram plot of gradient values for New Delhi image
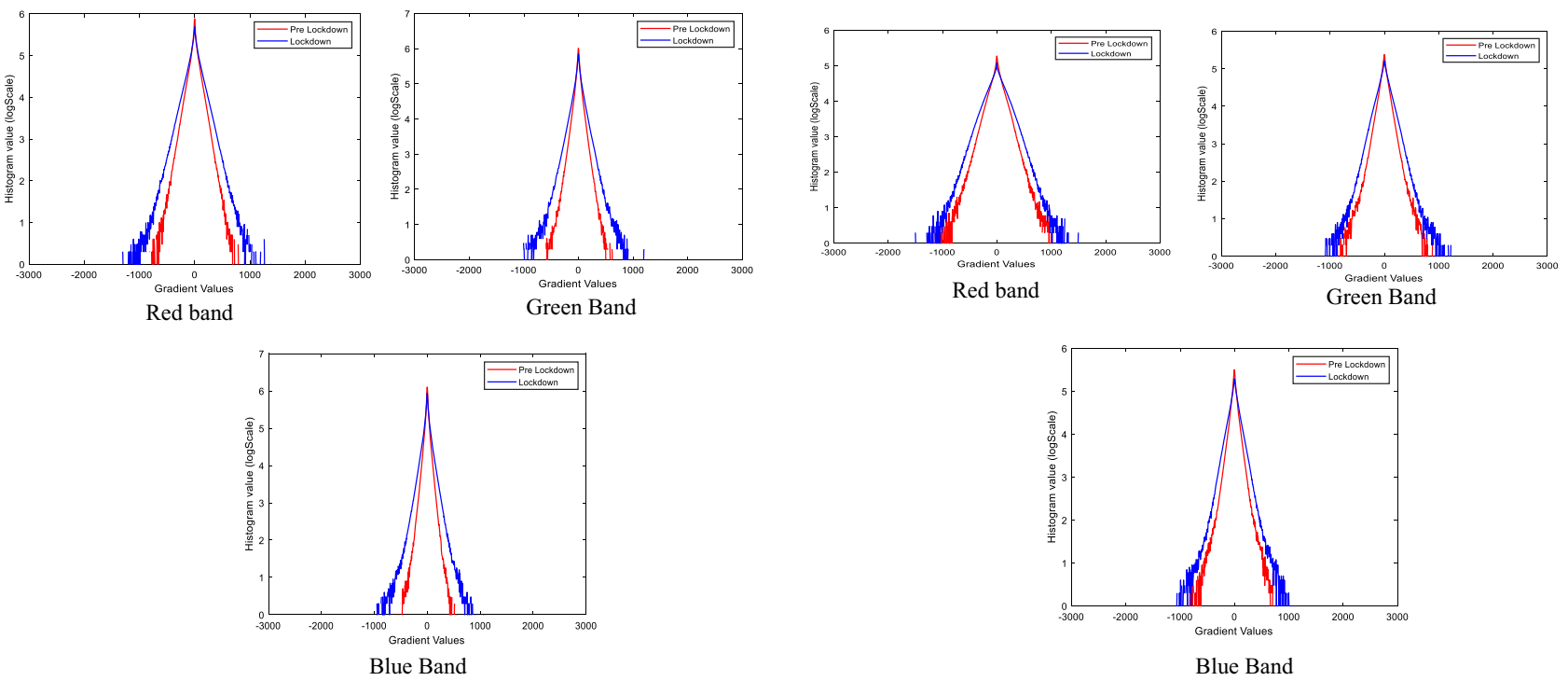

(c) Histogram plot of gradient values for Patna image

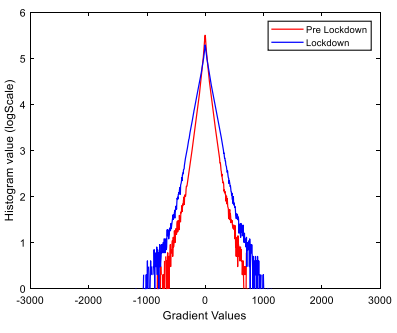

Blue Band

(d) Histogram plot of gradient values for Varanasi image

Fig. 13 Histogram plot of gradient values for images of different cities during pre-lockdown and lockdown period

improvement in the satellite images with respect to sharpness and reflectance values is observed due to a reduction in atmospheric effects in the satellite images. Since atmospheric effects are more prevalent in the blue band, hence improvement in image quality is observed more in the blue band as compared to the red and green band during the lockdown period. The power spectrum and the gradient method have been used to estimate the sharpness of the image. In both methods, frequency content in the lockdown period has increased and thus enhances the sharpness of the image. The reflectance values are also analysed for the images of the pre-lockdown and lockdown period. It is observed that the reflectance values for water, vegetation, and barren land (low reflectance values) are decreased in visible bands and reflectance values for building like structures (high reflectance values) are increased in visible bands during the lockdown period and thus contrast in the images has improved. On comparing pre-lockdown and lockdown datasets of the year 2020 with the similar dates in the year 2019, power spectrum analysis shows that atmospheric effects in the images are significantly reduced in the lockdown period in the year 2020 as compared to images of the similar dates in the year 2019 . 
Table 4 Reflectance values of different features

\begin{tabular}{|c|c|c|c|c|c|c|c|c|}
\hline \multirow[t]{2}{*}{ City } & \multirow[t]{2}{*}{ Reflectance $(\%)$} & \multicolumn{3}{|c|}{ Pre-lockdown period } & \multicolumn{3}{|c|}{ Lockdown period } & \multirow[t]{2}{*}{ Average percentage change } \\
\hline & & Red & Green & Blue & Red & Green & Blue & \\
\hline \multirow[t]{4}{*}{ New Delhi } & Building & 19.8 & 18.7 & 19.2 & 23 & 20.9 & 20.3 & 10.9 \\
\hline & Vegetation & 8.8 & 11.4 & 11 & 5.8 & 7.18 & 9.0 & -29.4 \\
\hline & Barren field & 18.2 & 15.3 & 15.3 & 18.5 & 14.4 & 13.1 & -6.3 \\
\hline & Water & 11.6 & 13.8 & 14.8 & 6.7 & 9.02 & 11.6 & -32.8 \\
\hline \multirow[t]{3}{*}{ Agra } & Building & 19.8 & 18.7 & 19.5 & 26.5 & 23.2 & 21.7 & 22.7 \\
\hline & Vegetation & 12.3 & 14.4 & 16.8 & 7.4 & 8.85 & 9.91 & -39.7 \\
\hline & Barren field & 13.4 & 14.3 & 16.3 & 10.8 & 10.8 & 11.3 & -24.9 \\
\hline \multirow[t]{4}{*}{ Patna } & Building & 26.2 & 23.1 & 23.2 & 35.4 & 31.1 & 29.4 & 32.0 \\
\hline & Vegetation & 11.7 & 13.8 & 15.4 & 11.7 & 13.1 & 13.3 & -6.5 \\
\hline & Barren field & 16.3 & 16.1 & 18 & 14.8 & 12.1 & 12.6 & -21.3 \\
\hline & Water & 13.6 & 15.5 & 17.1 & 9.1 & 11.9 & 12.2 & -28.4 \\
\hline \multirow[t]{3}{*}{ Varanasi } & Building & 23.4 & 20.4 & 18.9 & 27.1 & 23.1 & 20.1 & 11.6 \\
\hline & Vegetation & 8.97 & 12.7 & 13.3 & 7.95 & 11.8 & 11.6 & -10.4 \\
\hline & Water & 9.6 & 12.5 & 13.7 & 6.24 & 8.97 & 10.5 & -28.9 \\
\hline
\end{tabular}

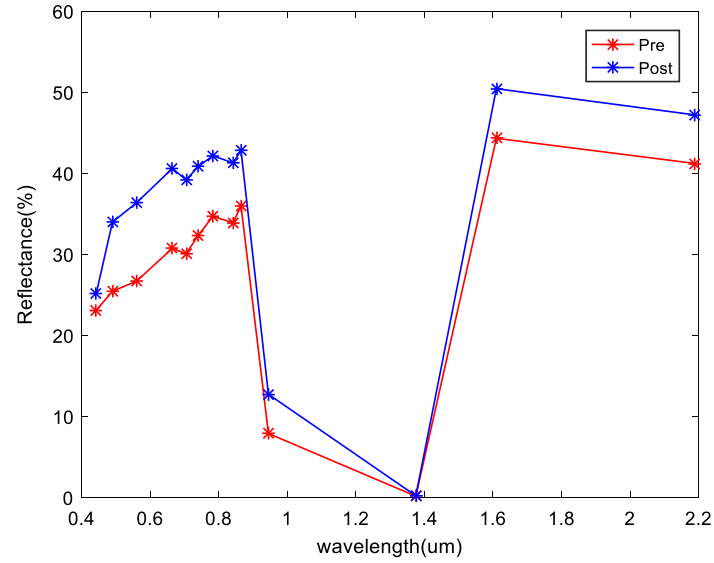

(a) Building

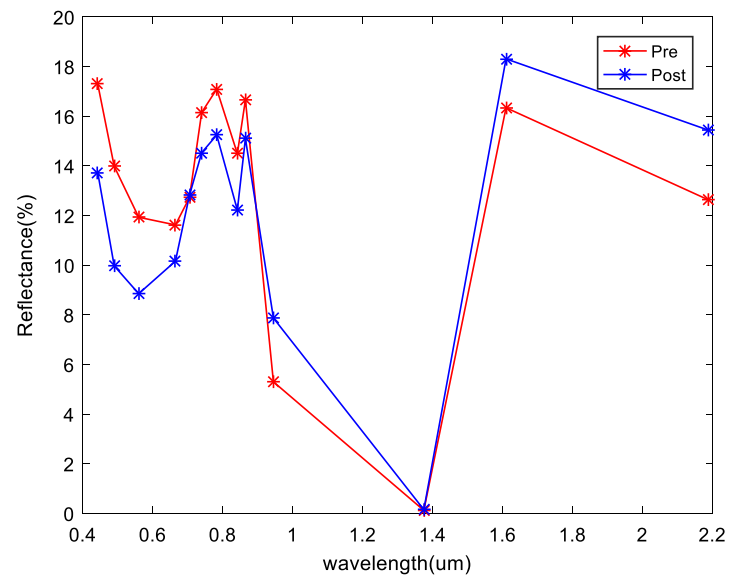

(c) Barren land

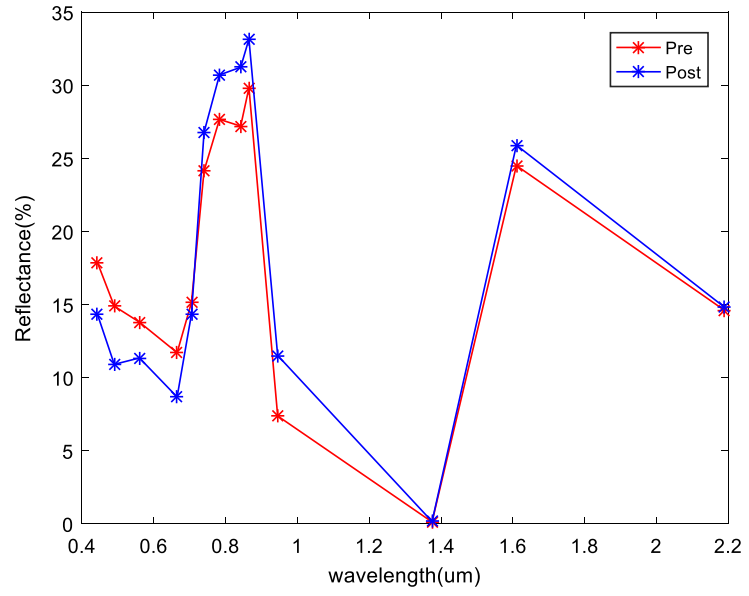

(b) Vegetation

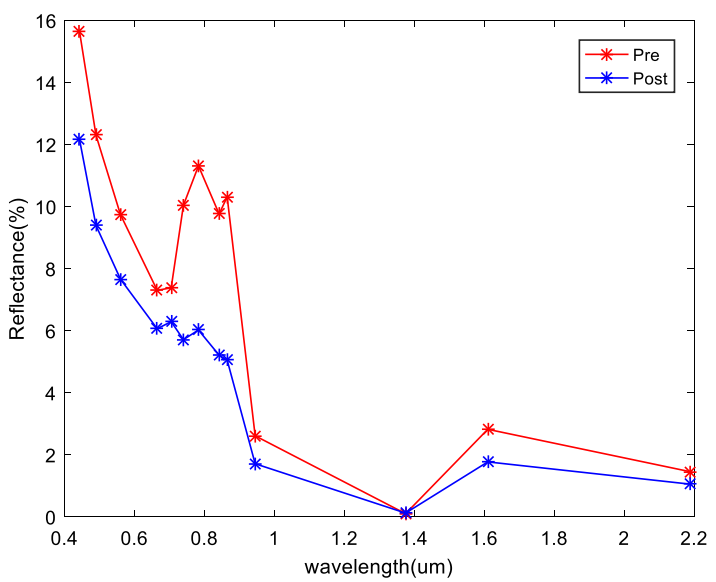

(d) Water

Fig. 14 Spectral profile of different features during pre-lockdown and lockdown period 


\section{References}

Bowker, D. E., Davis, R. E., Myrick, D. L., Stacy, K. J., \& William, T. (1985). Spectral reflectances of natural targets for use in remote sensing studies. NASA technical report RP-1139.

Dutheil, F., Baker, J. S., \& Navel, V. (2020). COVID-19 as a factor influencing air pollution? Environment Pollution, 263, 114466.

Eriksson, A. I. (2000). Spectral analysis. ISSI scientific report SR-001 ISSI/ESA.

Gatti, A., \& Bertolini, A. (2015). Sentinel-2 products specification document. ESA sentinel-2 PDGS. https://sentinel.esa.int/web/ sentinel/document-library/content/-/article/sentinel-2-level-1-tolevel-1c-product-specifications.Accessed April 12, 2020.

Guojun, H., Yuhang, P., \& Takanao, T. (2020). COVID-19, city lockdown, and air pollution: Evidence from China.https://www. medrxiv.org/content/10.1101/2020.03.29.20046649v2. Accessed April 12, 2020.

Kopeika, N. S., Sadot, D., \& Dror, I. (1998). Aerosol light scatter vs turbulence effects in image blur. In Proceedings of SPIE-the international society for optical engineering.
Michael, G., \& Brian, J. R. (2019). Image evolution using 2D power spectra. Hindawi Complexity. https://doi.org/10.1155/2019/ 7293193.

Ranjana, A., Kumar, P., \& Gorai, A. K. (2020). Effect of lockdown due to SARS COVID-19 on aerosol optical depth (AOD) over urban and mining regions in India. Science of the Total Environment. https://doi.org/10.1016/j.scitotenv.2020.141024.

Sifakis, N. (2000). Satellite image processing for haze and aerosol mapping (SIPHA): code description and presentation of results. In Conference: Geoscience and remote sensing symposium, 2000. Proceedings IGARSS 2000. IEEE 2000 international (Vol. 1).

Yoram, J. K. (1984). Atmospheric effects on remote sensing of surface reflectance. In Proceedings of the SPIE 0475, remote sensing: Critical review of technology.

Zenzo, S. (1986). A note on the gradient of a multi-image. Computer Vision, Graphics, and Image Processing, 33, 116-125.

Publisher's Note Springer Nature remains neutral with regard to jurisdictional claims in published maps and institutional affiliations. 